\title{
EFICACIA Y SEGURIDAD DE LA INYECCIÓN INTRAVÍTREA DE BEVACIZUMAB EN EL TRATAMIENTO DEL GLAUCOMA NEOVASCULAR: REVISIÓN SISTEMÁTICA
}

\author{
EFFICACY AND SAFETY OF INTRAVITREAL INJECTION OF \\ BEVACIZUMAB IN THE TREATMENT OF NEOVASCULAR \\ GLAUCOMA: SYSTEMATIC REVIEW
}

\author{
MARTÍNEZ-CARPIO PA ${ }^{1}$, BONAFONTE-MÁRQUEZ E², HEREDIA-GARCÍA CD ${ }^{3}$, \\ BONAFONTE-ROYO $\mathrm{S}^{2}$
}

\section{RESUMEN}

Propósito: Revisión sistemática sobre la eficacia y seguridad de la inyección intravítrea de bevacizumab (IVB) en el tratamiento del glaucoma neovascular (GNV). Se incluyen todos los trabajos originales publicados en Medline hasta agosto de 2008. Métodos: Búsqueda y selección de la información en Internet y Medline, validadas mediante el Índice Kappa (K). Estudio estadístico y clínico de los resultados en los trabajos seleccionados, uno a uno. Resultados: Se encuentran 26 artículos originales que analizan la eficacia y seguridad del procedimiento en casos únicos y en series cortas de casos. 24 de ellos se analizan conjuntamente, con un total de 127 ojos intervenidos. La eficacia calculada para casos difíciles es del $68,7 \%$. Las recidivas en un seguimiento de 4,2 meses son del $18,6 \%$. Todos los trabajos son posteriores a 2006 y ninguno cumple criterios de ensayo clínico aleatorizado. Las com-

\begin{abstract}
Purpose: Systematic review on efficacy and safety of intravitreal bevacizumab (IVB) in the treatment of neovascular glaucoma (NVG). All original papers published in Medline (prior to August 2008) were included.

Methods: Search and selection of information on the internet and in Medline, validated by Kappa Index (K). Statistical and clinical study of the results in the selected articles on a one by one basis. Results: 26 original papers analyzed the efficacy and safety of the procedure in case reports and short series of cases (127 eyes). The efficacy calculated in the sample was $68.7 \%$ and the recurrence rate was $18.6 \%$ in 4.2 months of follow-up. All studies were after 2006 and none of them was a clinical randomized controlled assay. Ophthalmic complications were under $0.78 \%$ and no systemic complications were found.
\end{abstract}

\footnotetext{
Recibido: 6/2/08. Aceptado: 16/9/08.

1 Unidad de Investigación Ocular y Láser. Centro de Oftalmología Bonafonte. Barcelona. Departamento de Investigación Láser. IMCInvestiláser. Sabadell. Barcelona. España.

2 Departamento de Cirugía Ocular y Láser. Centro de Oftalmología Bonafonte. Barcelona. España.

3 Departamento de Retina y Vítreo. Centro de Oftalmología Bonafonte. Barcelona. España.
} 
plicaciones de la IVB son inferiores al $0,78 \%$ y no se observan complicaciones sistémicas.

Conclusiones: El bevacizumab demuestra que las inyecciones intravítreas pueden ser muy eficaces y seguras para la manipulación terapéutica de los factores de crecimiento en el segmento anterior. $\mathrm{La}$ IVB se perfila como un tratamiento de primera línea en casos de GNV dificiles. Se necesitan ensayos clínicos controlados que confirmen estos resultados y autorizen el uso.

Palabras clave: Bevacizumab, avastin, inyección intravítrea, glaucoma neovascular, rubeosis iris, fotocoagulación láser, factores de crecimiento, revisión sistemática.
Conclusions: The use of bevacizumab demonstrates that intravitreal injections may be effective and useful to manipulate growth factors in the anterior chamber. IVB could serve as a first line treatment for NVG. Clinical trials are needed to confirm these results before its use is authorized (Arch Soc Esp Oftalmol 2008; 83: 579-588).

Key words: Bevacizumab, avastin, intravitreal injection, neovascular glaucoma, rubeosis iridis, laser photocoagulation, growth factors, systematic review.

\section{INTRODUCCIÓN}

En la especie humana el Factor de Crecimiento Endotelial Vascular (hVEGF), el Factor de Crecimiento Epidérmico (hEGF) y el Factor Transformante del Crecimiento-beta (hTGF-beta), junto a sus receptores de membrana (hVEGFR, hEGFR y hTGF-betaR) juegan un papel crucial, ocasionalmente antagónico o sinérgico, en el control de la proliferación celular de los tejidos sanos y tumorales y en la neoformación vascular secundaria a dicha proliferación. Las investigaciones realizadas en cultivos celulares de estas moléculas ha sido muy eficaz para descubrir los mecanismos de regulación entre ellas. La síntesis de anticuerpos monoclonales contra estos factores y sus receptores arrojará alternativas terapéuticas muy efectivas para tratar los procesos neoformativos en los próximos años (1-7).

En 2006 aparecen las primeras publicaciones que evalúan la eficacia y seguridad del bevacizumab (Avastin, Genentech) en el tratamiento del glaucoma neovascular (GNV). El bevacizumab es un anticuerpo monoclonal dirigido contra todas las isoformas biológicamente activas del VEGF-A humano (hVEGF-A), uno de los estímulos angiogénicos más importantes $(8,9)$. Se trata del primer anticuerpo anti-VEGF aprobado por la Food and Drug Administration (FDA) como tratamiento de administración intravenosa en pacientes con cáncer (8).

El GNV comienza con el crecimiento de una fina membrana fibrovascular sobre la superficie del ángulo iridocorneal. Cuando dicha membrana cubre un área importante del trabéculo, se obstaculiza el drenaje del humor acuoso y se produce un glauco- ma de ángulo abierto. En fases más avanzadas el tejido fibrovascular se contrae, el iris se arrastra hasta el ángulo camerular con una extensa goniosinequia iridiana que acaba en un glaucoma de ángulo cerrado. No obstante, la simple presencia de neovasos en el ángulo, incluso por células y exudados liberados a nivel microcirculatorio, puede acabar en el mismo problema, de pronóstico grave por las lesiones isquémicas renitianas previsibles (10).

A mediados de 2007 algunos expertos plantearon si el uso de bevacizumab era un tratamiento «compasivo» o una manera de ganar dinero para los oftalmólogos que lo utilizan (11). Se trata de un fármaco cada vez más utilizado en oftalmología, pero todavía no autorizado. Su bajo coste respecto a otros inhibidores del VEGF parece influir negativamente en su comercialización y aprobación para uso intravítreo, cuando las evidencias científicas sugieren que su perfil de eficacia y seguridad a dosis de 1-2 mg/dL en vítreo parece excelente para numerosas indicaciones. En nuestro Centro desde 2006 hemos tratado seis casos de GNV con bevacizumab. La respuesta a corto y medio plazo ha sido excelente, coincidiendo con las publicaciones, pero se necesita una cobertura científica para justificar su uso en esta indicación. De momento el tratamiento del GNV mediante inyección intravítrea de bevacizumab (IVB) se basa en resultados experimentales de laboratorio y en una experiencia clínica bastante limitada.

El propósito de este trabajo es estudiar los datos de eficacia y seguridad de la IVB en el GNV, según los datos aparecidos en las revistas especializadas hasta agosto de 2008. La revisión sistemática y el meta-análisis aportan la mejor evidencia disponible 
sobre un tema de interés, si bien una lectura objetiva y crítica de cada trabajo es indispensable para discutir los resultados. Se suele hablar de revisión sistemática para referirse al proceso de identificar sistemáticamente y evaluar varios estudios del mismo tipo y con un objetivo común. El meta-análisis consiste en utilizar técnicas estadísticas que combinan los resultados de un grupo homogéneo de estudios con medidas globales en la cuantificación y calidad de resultados (12-15). Aquí se intentan ambas posibilidades.

\section{SUJETOS, MATERIAL Y MÉTODOS}

La búsqueda, selección y cuantificación de resultados se realizó de por dos investigadores independientes (PMC y EBM), con el siguiente consenso preestablecido:

\section{Estrategia de búsqueda}

Se acude a la base de datos Medline y a la red Internet. Para obtener datos de eficacia y seguridad de la IVB en el GNV en Medline, entre enero de 2003 y octubre de 2008, se utilizaron las opciones de escrutinio recomendadas por los expertos (1214). Se tantearon diferentes palabras clave, descriptores compuestos y meta-términos en orden creciente de especificidad hacia el tema estudiado, seleccionando y cruzando los más productivos y selectivos (12-14). Se leyeron los resúmenes de todos los trabajos y se recopilaron todos los artículos completos incluidos en el estudio. La búsqueda se complementó con un vaciado en Internet (Firefox-Google) al efecto de encontrar publicaciones científicas fiables omitidas en Medline $(14,15)$.

\section{Selección de artículos para el análisis}

Criterios de inclusión:

- El objetivo fundamental del trabajo es valorar la eficacia y seguridad de la IVB en el tratamiento del GNV.

- El estudio aporta datos necesarios de la historia clínica oftalmológica y detalla el problema ocular antes de la intervención.

- Los materiales y métodos permiten reproducir la investigación.
- Se especifica cuándo y cómo se observan los resultados, aportando pruebas objetivas.

- Se trata de una publicación reconocida por un organismo científico.

Criterios de exclusión:

- Artículos sobre la eficacia de la IVB en procesos de neoformación vascular intraocular, incluyendo de manera accesoria el GNV, sin datos de eficacia, o que se prestan a confusión.

- Comunicaciones en congresos, experiencias de investigadores no publicadas, opiniones de autor, etc.

\section{Estadística descriptiva de agrupación}

Se considera el número de ojos intervenidos en cada estudio (n), el tiempo de seguimiento máximo tras la IVB, en meses (t) y la pérdida o persistencia de eficacia durante los seguimientos. En todo el análisis se utilizan como indicadores descriptivos la media aritmética $(\mathrm{m}=$ promedio $)$ como medida de

Tabla I. Análisis agrupado de datos en $\mathbf{2 4}$ publicaciones seleccionadas. Se indica el promedio y entre paréntesis el rango

\begin{tabular}{|c|c|c|c|c|c|}
\hline Bibliografía & $\mathrm{n}$ & $\mathrm{EC}$ & $\mathrm{ECP}$ & ECM & Seguimiento \\
\hline Gheith (18) & 6 & 2,5 & 15 & 18 & $9,7(*)$ \\
\hline Kelkar (19) & 1 & 3 & 3 & 3 & 1,5 \\
\hline Yazdani (20) & 2 & 3 & 6 & 6 & 1,5 \\
\hline Chilov (21) & 3 & 2,6 & 7,8 & 9 & 1,5 \\
\hline Batioglu (22) & 1 & 2 & 2 & 3 & 0,25 \\
\hline Vatavuk (23) & 1 & 3 & 3 & 3 & 1 \\
\hline Jonas (24) & 2 & 2 & 4 & 6 & 3 \\
\hline Iliev (25) & 6 & 2 & 12 & 18 & 4 \\
\hline Mason (26) & 3 & 2,6 & 7,8 & 9 & 0,75 \\
\hline Silva (27) & 1 & 3 & 3 & 3 & 2 \\
\hline Grisanti(28) & 6 & 2,6 & 15,6 & 18 & 1 \\
\hline Kahook (29) & 1 & 3 & 3 & 3 & 1,5 \\
\hline Davidorf (30) & 1 & 3 & 3 & 3 & 2 \\
\hline Avery (31) & 1 & 3 & 3 & 3 & 0,25 \\
\hline Oshima (32) & 7 & 2,7 & 18,9 & 21 & 2 \\
\hline Aswad (33) & 2 & 2 & 4 & 6 & 4 \\
\hline Wakabayashi (34) & 17 & 2,4 & 40,8 & 51 & $13,3(*)(1)$ \\
\hline Wakabayashi (34) & 15 & 1,3 & 19,5 & 45 & $13,3(*)(2)$ \\
\hline Ehlers (35) & 23 & 2,5 & 57,5 & 69 & $3,2(*)$ \\
\hline Chalam (36) & 16 & 2,3 & 36,8 & 48 & 1 \\
\hline Kitnarong (37) & 6 & 2,5 & 15 & 18 & 6 \\
\hline Cheng (38) & 3 & 3 & 9 & 9 & 1 \\
\hline Cornish (39) & 2 & 3 & 6 & 6 & 6 \\
\hline Sothornwit (40) & 1 & 3 & 3 & 3 & 3 \\
\hline
\end{tabular}

$\Sigma \mathrm{n}=127 ; \mathrm{EC}=2,59(\mathrm{r}=2-3) ; \Sigma \mathrm{ECP}=262 ; \mathrm{ECM}=3 \mathrm{n}=381 ;$ $\mathrm{EG}=248 / 360=68,9 \% ; \mathrm{t}=4.2(\mathrm{r}=0,25-13,3)$ (tiempo máximo de seguimiento, en meses); (*) t promedio de seguimiento; GNV ángulo abierto (1); GNV ángulo cerrado (2). 
tendencia central, y el recorrido $(\mathrm{r}=$ rango) como grado de dispersión.

Para valorar la eficacia clínica del tratamiento (EC) en cada artículo se establece la siguiente escala semicuantitativa: empeoramiento [-1], sin eficacia [0], eficacia moderada [1], eficacia buena [2], eficacia excelente [3]. Para puntuar cada trabajo se tuvieron en cuenta los resultados de las pruebas diagnósticas y el resto de tratamientos aplicados, además de la IVB.

Se anotó para cada trabajo la dosis del fármaco administrada, posible fotocoagulación antes y/o después de la IVB, necesidad de cirugía filtrante, tratamientos médicos y convencionales aplicados, porcentaje de recidivas y complicaciones observadas. También los datos más destacados de la discusión y conclusión de cada trabajo.

Las puntuaciones de la EC se pautaron previamente por ambos investigadores. Cuando los trabajos estudiaban series de casos, con resultados para cada caso, se tomó el valor promedio. Se define como eficacia clínica ponderada (ECP) el producto EC x n, y eficacia global, o grupal (EG), al cociente ECP/ECM, donde ECM es la eficacia clínica máxima (=3n).

\section{Validación de los resultados}

Para validar la fiabilidad interobservador en la búsqueda, selección de artículos y puntuación de eficacia en la escala semicuantitativa se calculó el índice Kappa $(\mathrm{K})$. Se consideró concordancia válida si $\mathrm{K}>0$,7. Las discrepacias entre ambos investigadores se anularon por consenso utilizando el razonamiento crítico-deductivo $(16,17)$.

\section{RESULTADOS}

Resultados de la búsqueda en MEDLINE 2003enero de 2008:

(O: artículos originales / R: revisiones).

Avastin/Bevacizumab (O1167/R371).

Avastin/Bevacizumab/ophthalmology/eye therapy (O199/R15).

Avastin/Bevacizumab/neovascular glaucoma (O19/R2).

Avastin/Bevacizumab/treatment neovascular glaucoma (O18/R2).

Resultados de la búsqueda en MEDLINE (2003octubre de 2008):
(O: artículos originales / R: revisiones).

Avastin/Bevacizumab (O1991/R739).

Avastin/Bevacizumab/neovascular glaucoma (O37/R4).

Avastin/Bevacizumab/treatment neovascular glaucoma (O28/R4).

Resultados de la búsqueda en INTERNET (Octubre 2008): Aparecen 30900 entradas para Bevacizumab/Glaucoma. Se encuentran 14 publicaciones y comunicaciones procedentes de organismos y sociedades, que no aparecían en Medline.

Los índices kappa de concordancia interobservador fueron: búsqueda en Medline $(\mathrm{K}=1)$, búsqueda en Internet $(K=0,83)$, selección de trabajos $(K=0,92)$ y valoración eficacia clínica $(K=0,80)$. De 37 artículos seleccionados, 26 cumplían criterios de inclusión (18-42). Para el análisis agrupado se acordó excluir el estudio de Bianciotto porque la causa del GNV era tumoral y la respuesta al fármaco podría ser diferente (41). También se elimina el trabajo de Avery y cols. (42) porque los pacientes con GNV no se diferencian bien de los que sólo tienen lesiones en retina. El artículo de Wakabayashi (34) se divide en dos partes para diferenciar entre GNV de ángulo abierto o cerrado y homogeneizar la eficacia clínica según la severidad del glaucoma. Las características de los trabajos imposibilitaron el meta-análisis: ninguno cumple criterios de ensayo clínico controlado y son heterogéneos en la selección de pacientes, en las indicaciones y aplicación del procedimiento y en los tratamientos concomitantes que se utilizan. Son estudios retrospectivos que no incluyen datos estadísticos manipulables en el meta-análisis convencional. Sin embargo, algunas variables importantes son susceptibles de un análisis descriptivo y crítico del máximo interés. Los 24 trabajos sometidos a este análisis de integración de datos reflejan fielmente el estado actual de conocimiento en esta materia. Las variables promediables incluyen la dosis utilizada, las alteraciones gonioscópicas y clínicas encontradas y todos los tratamientos médicos y quirúrgicos que se utilizan para solventar el caso, además de la IVB.

\section{Estadística descriptiva y resultados clínicos en los trabajos seleccionados}

Todos aparecen en Medline, excepto uno (33). No se encuentran publicaciones previas a 2006, el $38 \%$ son de 2006 , el $29 \%$ de 2007 y el $33 \%$ de ene- 
ro a agosto de2008. El $79 \%$ de los trabajos estudian series cortas de casos con menos de 6 ojos intervenidos. El mejor estudio y el más amplio es el de Wakabayashi (34), que trata 41 ojos, 9 con rubeosis iridis sin glaucoma, 17 con GNV de ángulo abierto y 15 con GNV de ángulo cerrado. También destacan por el tamaño muestral los artículos de Ehlers $(n=23)$ y Chalam $(n=16)$. Los estudios aparecidos en 2008, de mayor calidad, incluyen el $65 \%$ de ojos analizados y aclaran algunas las incógnitas planteadas entonces.

La eficacia clínica global (EG) calculada en 127 ojos con un seguimiento máximo promedio de 4,2 meses fue del $68,7 \%$ y el porcentaje de recidivas del $18,6 \%$. La respuesta a una segunda dosis de tratamiento fue efectiva en todos los casos de recidiva. La dosis de bevacizumab más utilizada fue $1,25 \mathrm{mg} / 0,05 \mathrm{~mL}(\mathrm{~m}=1,29 \mathrm{mg}, \mathrm{r}=1-2,5 \mathrm{mg})$. Las complicaciones oftalmológicas se sitúan por debajo del $0,78 \%$ (1 ojo), sin demostración de causalidad. No se detecta ninguna complicación sistémica.

En la muestra estudiada aquí, las causas principales de GNV fueron la retinopatía diabética y la oclusión de la vena central de la retina, incluyendo dos casos de oclusión de la arteria central de la retina $(23,26)$, un caso de meningioma del ápex orbitario tratado con radioterapia (21), un caso de paciente diabético con melanoma coroideo (30) y un melanoma de iris tratado con radioterapia (41). Generalmente se justificó el uso de la IVB por el fracaso de tratamientos previos o por un abordaje difícil.

Todos los ojos tratados en los que el glaucoma es inexistente o mínimo, la eficacia de la IVB para reducir la rubeosis iridis y angular es del 100\% (demostrado angiográficamente), y del $85 \%$ para eliminarla, independientemente de la etiología. En más del $80 \%$ de los ojos tratados antes de 2008 la neovascularización asociada a glaucoma desaparece completamente, la PIO se normaliza en pocos días sin necesidad de cirugía filtrante y mejora la agudeza visual. Cuando se considera conveniente cirugía del glaucoma, tras la IVB, o al mismo tiempo, se produce menos sangrado y mejora en confort del paciente $(24,26,37,40)$. El tratamiento con IVB no parece suficiente en caso de sinequias periféricas anteriores consolidadas $(18,25)$ y cuando existe cierre pronunciado del ángulo (34).

Faltan estudios a medio y largo plazo para poder concretar cifras. El efecto farmacológico es drástico y muy precoz a la visualización gonioscópica.
Los efectos se observan a las 48 horas, con un rango entre las 24 horas y la primera semana $(23,25-$ 29,32,33,37-39). En los 127 ojos intervenidos sólo se detecta un caso de queratitis infecciosa, sin demostración de causalidad (21) y ninguna complicación sistémica. La dosis de $1 \mathrm{mg}$, incluso inferior, podría ser igual de efectiva que 1,25 mg para tratar el GNV, quizá reduciendo las recidivas $(26,28,29,34)$. En cambio la dosis de 2,5 mg parece facilitarlas (20) y conviene desaconsejarla en esta indicación. Cuando ha sido necesario una segunda IVB tampoco aparecen complicaciones y se logra resolver el caso $(18,20,32)$. La mayoría de pacientes se trataron con fotocoagulación panretiniana antes y/o después de la IVB, evitando generalmente la ciclofotocoagulación transescleral y la cirugía filtrante. Los resultados también son satisfactorios cuando falla la panfotocoagulación y ciclofotocoagulación previa (29).

Las recidivas hasta 2008 aparecen en el 16\% de casos y ocurren generalmente entre el segundo y quinto mes $(18,20,32)$. No obstante, el dato es poco orientativo porque muchos seguimientos son de menos de dos meses. Hasta entonces, sólo 11 ojos se examinan consecutivamente más allá de los 3 meses, recidivando el 27\%. En 2008 Wakabayashi y Ehlers aclaran la veracidad de este resultado, en muestras más amplias. Pacientes con rubeosis iris sin glaucoma en los que desaparecen los neovasos podrían tener hasta el $40 \%$ de recidivas durante los primeros seis meses, pero todas se resuelven satisfactoriamente con una segunda IVB (34). Las recidivas precoces (antes de 1 mes) podrían aparecer en más del $12 \%$ de casos (36). El promedio de recidivas calculado en este trabajo es del $18,6 \%$ en un seguimiento promedio de 4,2 meses.

En casos de rubeosis iridis y glaucoma mínimo la IVB podría ser eficaz, por sí sola, para tratar el problema. Cuando la tracción del ángulo es significativa, la eficacia depende de si es abierto o cerrado. En el GNV abierto la IVB podría ser eficaz en más del $70 \%$ de casos, evitando muchos casos de cirugía. En cambio, cuando el ángulo es cerrado más del 90\% de casos necesitan cirugía (34). Ehlers también confirma las investigaciones previas comprobando las ventajas de la IVB asociada a la fotocoagulación panretiniana, respecto a la fotocoagulación panretiniana sola. En un seguimiento de 4 meses, la regresión neovascular fue mayor, y más precoz, en el grupo tratado con ambos métodos (35). Los casos de GNV debidos a tumores también 
respondieron positivamente, pero merecen un estudio a parte $(21,23,26,41)$. No se han estudiado los efectos de la IVB como tratamiento único.

En 24 estudios diferentes (127 ojos) se encuentra un beneficio/riesgo muy favorable a la IVB para tratar el GNV. En casos de neovascularización en iris y ángulo sin glaucoma, y en casos incipientes de glaucoma, la IVB per se podría ser resolutiva. Además, al evitar el riesgo de hemorragia, mejora el pronóstico del GNV tratado con fotocoagulación y/o cirugía. Existe acuerdo común en que la IVB aporta como valor añadido una regresión precoz de los neovasos y una normalización de la PIO mejor y más rápida. Algunos trabajos sugieren que el éxito del tratamiento repercute en una mejora de la agudeza visual, pero no pueden demostrarlo con la metodologia que utilizan. Varios autores apuntan que la IVB es capaz de controlar la PIO en GNV refractarios al tratamiento, en los términos de Sothornwit (40).

\section{DISCUSIÓN}

Los primeros trabajos de Linch (9) y Rosenfeld (8) sugieren amplias posibilidades de la IVB a nivel farmacológico para favorecer la regresión de las neoformaciones vasculares. Entre los estudios clínicos que aparecen en Medline destaca el de Avery (34) en un total de 45 ojos intervenidos para un grupo heterogéneo de proliferaciones vasculares intraoculares. Los resultados de eficacia fueron del $73 \%$ en retina y del $82 \%$ en iris. A las 24 horas postinyección ya se aprecian signos de involución neovascular en todos los pacientes tratados, sin ningún efecto secundario oftalmológico ni sistémico, mantenido al menos durante 11 semanas. Nuestros resultados apoyan la seguridad y rapidez del efecto, con un éxito más modesto $(68,7 \%)$ porque trata numerosos glaucomas establecidos de difícil respuesta. Las publicaciones más recientes corroboran los resultados previos, minimizando el sesgo de publicación conocido como fenómeno iceberg. Los 26 trabajos seleccionados, y los 24 analizados, concluyen que la IVB tiene un claro valor terapéutico añadido al resto de tratamientos, mostrándose eficaz y sin complicaciones. El beneficio/riesgo es muy favorable y cabe plantear la IVB y la panfotocoagulación retinal como tratamiento inicial del GNV, independientemente de si se decide o no cirugía filtrante. Cuando el GNV se asocia a hemorra- gia vítrea, ésta puede resolverse con éxito. Así lo notifican algunos medios e interlocutores en Internet, que no se valoran aquí. Los resultados coinciden con los publicados en revistas con factor de impacto y sugieren que la práctica clínica experimental de la intervención es muy superior a la que se comunica a la literatura.

El modo oficial y válido para estudiar la eficacia y seguridad de un fármaco es el ensayo clínico controlado y aleatorizado en fases II-IV. Actualmente la información procede de series retrospectivas cortas de casos que no cumplen criterios de ensayo clínico para el estudio farmacológico. Los artículos originales que se revisan plantean como finalidad comprobar si el bevacizumab intravítreo es eficaz y seguro en el tratamiento del GNV, en cambio los diseños y la metodología que emplean no permiten demostrar, sólo sugerir. Esta revisión aclara la necesidad de priorizar los ensayos clínicos pertinentes dada la gravedad del GNV y los buenos resultados que observan las investigaciones clínicas preliminares.

En el caso del GNV el bevacizumab podría ser más efectivo que el resto de inhibidores del VEGF comercializados, como ranibizumab y pentaganib, por sus características moleculares. La ventaja propuesta para ranibizumab, como molécula de menor tamaño y más apropiada para penetrar en retina, no se justifica en segmento anterior. El bevacizumab es posiblemente el fármaco mejor estudiado para el tratamiento intravítreo del GNV, tanto en el laboratorio como en los pacientes. Se acaba de comprobar que en casos de rubeosis iridis y GNV las concentraciones de VEGF en el humor acuoso están aumentadas y que se reducen drásticamente con la IVB (43). Nuestros resultados sugieren que la eficacia de la IVB para resolver el glaucoma depende de las lesiones estructurales del trabéculo y de la fisiopatología relacionada con la membrana fibrovascular que se desarrolla en la superficie del ángulo iridocorneal. Esta membrana provoca, en un primer tiempo, un GNV de ángulo abierto, que puede desaparecer con la IVB. Cuando la membrana se fibrosa y se transforma en una goniosinequia iridiana, cierra traccionalmente el ángulo y suele necesitarse cirugía filtrante. La IVB elimina los neovasos, pero no es capaz de deshacer el tejido fibroso y la sinequia. En este proceso además del VEGF intervienen otros factores de crecimiento.

La mayor importancia de este trabajo es que demuestra la capacidad que tienen las inyecciones intravítreas para modificar factores de crecimiento 
en cámara anterior, con resultados en pacientes que confirman los estudios de laboratorio. La ventaja es que dosis muy pequeñas del fármaco atraviesan bien los medios y alcanzan concentraciones efectivas en los lugares diana. Se demuestra que los factores de crecimiento funcionantes en el trabéculo y en el humor acuoso pueden manipularse farmacológicamente con las inyecciones intravítreas. Las posibilidades de futuro son importantes, si se piensa en los factores de crecimiento señalados en la introducción (1-6). Aunque la eficacia de bevacizumab en iris, coroides y retina parece evidente, no se puede concretar si los beneficios de esta molécula dependen exclusivamente del bloqueo o inactivación del VEGF o de otros factores de crecimiento y citoquinas, regulados por el VEGF, que desencadenan complejas cascadas de señalización intracelular (44). Otros factores de crecimiento celular pueden ser más importantes para disminuir la fibrosis o la proliferación de ciertas células. En cultivos de la línea tumoral humana MDA-MB-231 se han demostrado interacciones importantes entre los factores VEGF, EGF y TGF-beta, que también se han visto en células epiteliales, endoteliales e inmunitarias tanto en tejidos sanos como cancerosos (1-4). El humor acuoso y el trabéculo son muy ricos en factores de crecimiento y en moléculas y células relacionadas con los procesos inflamatorios e inmunes (45). Los más importantes, a nuestro criterio, son el VEGF, EGF y TGF-beta (45-48). Por dos motivos: los tres tienen una implicación crucial en la etiopatogenia del GNV y pueden manipularse simultáneamente con inyecciones intravítreas que combinen fármacos e inmunofármacos. Existen activadores e inhibidores del EGF y el TGF-beta, que operan directamente sobre ellos o sobre sus receptores de membrana. En la proliferación celular en las lentes intraoculares tienen un papel muy destacado la vías MAPK/ERK1/2 y ERK1/2, relacionadas con los receptores tirosina-quinasa, con el EGF y con sus receptores (EGFR). Los inhibidores farmacológicos que ya existen podrían ser efectivos en administración intravítrea para prevenir o deshacer procesos de epitelización, pero falta experimentarlo en animales $(44,46)$. También se comprueba que el TGF-beta determina la cantidad y composición de la matriz en la región yuxtacanalicular del trabéculo, y que, igual que en el caso del VEGF, las concentraciones de TGF-beta en el humor acuoso están aumentadas cuando hay glaucoma. La proteina BMP-7 en el laboratorio se comporta como un potente inhibidor de la fibrinogénesis mediada por el TGF-beta en las células trabeculares (45), y su combinación con bevacizumab en animales de experimentación tampoco se ha comprobado.

En el GNV los inhibidores del VEGF podrían solventar el problema vascular, mientras que los inhibidores o potenciadores del EGF y TGF-beta podrían actuar sobre la formación de la membrana fibrovascular y su progresiva retractilidad. Es decir, sobre la estructura responsable del glaucoma y la que determina el tipo de glaucoma en función del tiempo de evolución. La inyección intravítrea en cocktail podría conseguir normalizar la vascularización e interrumpir o regresar la formación de membranas y sinequias.

La importancia del bevacizumab en el tratamiento del GNV sobrepasa el interés particular de esta enfermedad. Tiene estrecha relación con algunas publicaciones aparecidas en esta revista en segmento anterior $(48,49)$. La uveítis anterior es una de las manifestaciones más importantes de la artritis juvenil idiopática. Entre las complicaciones aparece glaucoma, catarata y alteraciones corneales. Los factores de crecimiento antes indicados pueden jugar un papel importante, siendo las inyecciones intravitreas un abordaje efectivo y seguro para conseguir efectos farmacológicos, a dosis bajas, en todo el segmento anterior (48). El láser de titanio zafiro es capaz de producir en el conejo una trabeculoplastia , con mínimo o nulo efecto térmico, sólo con la actividad biomoduladora propia de la terapia láser de baja potencia. Los factores de crecimiento y sus receptores son especialmente sensibles a este tipo de estimulación, donde predominan los efectos fotoquímicos (49). Son líneas de investigación aparentemente desconexas donde los factores de crecimiento celular deben jugar una papel predominante.

\section{BIBLIOGRAFÍA}

1. Martínez-Carpio PA, Mur C, Rosel P, Navarro MA. Constitutive and regulated secretion of epidermal growth factor and transforming growth factor-betal in MDA-MB-231 cell line in 11-day cultures. Cell Signal 1999; 11: 753-757.

2. Martínez-Carpio PA, Mur C, Fernández-Montolí ME, Ramon JM, Rosel P, Navarro MA. Secretion and dual regulation between epidermal growth factor and transforming growth factor-betal in MDA-MB-231 cell line in 42 hour-long cultures. Cancer Lett 1999; 147: 25-29.

3. Masuda M, Suzui M, Lim JT, Deguchi A, Soh JW, Weinstein IB. Epigallocatechin-3-gallate decreases VEGF production in head and neck and breast carcinoma cells by 
inhibiting EGFR-related pathways of signal transduction. J Exp Ther Oncol 2002; 2: 350-359.

4. Donovan D, Harmey JH, Toomey D, Osborne DH, Redmon HP, Bouchier-Hayes DJ. TGF beta-1 regulation of VEGF production by breast cancer cells. Ann Surg Oncol 1997; 4: 621-627.

5. Valente $S$, Souto B, Balter $H$, Welling MM, Román E, Robles A, et al. Interaction of a monoclonal antibody against hEGF with a receptor site for EGF. Nucl Med Biol 1999; 26: 937-942.

6. Liss C, Fekete MJ, Hasina R, Lam CD, Lingen MW. Paracrine angiogenic loop between head-and-neck squamouscell carcinomas and macrophages. Int J Cancer 2001; 93: 781-785.

7. Presta LG, Chen H, O'Connor SJ, Chisholm V, Meng YG, Krummen L, et al. Humanization of an anti-vascular endothelial growth factor monoclonal antibody for the therapy of solid tumors and other disorders. Cancer Res 1997; 57: 4593-4599.

8. Rosenfeld P. An update on bevacizumab. Rev Ophthalmol 2005; $12: 12$.

9. Lynch SS, Cheng CM. Bevacizumab for neovascular ocular diseases. Ann Pharmacother 2007; 41: 614-625.

10. Bonafonte $S$, Arévalo F, García RA. Neovascularización del iris y glaucoma neovascular. En: Bonafonte S, García ChA. Retinopatía Diabética. II Ed. Madrid: Elsevier España, 2006.

11. Spratt A, Ogunbowale L, Franks W. Bevacizumab: a compassionate way of doing business? Eye 2007; 21: 890-891.

12. Dickersin K, Scherer R, Lefevre C. Identifying relevant studies for systematic reviews. BMJ 1994; 309: 1286-1291.

13. Helmer D, Savoie I, Green C, Kazanjian A. Evidence-based practice: extending the search to find material for the systematic review. Bull Med Libr Assoc 2001; 89: 346-352.

14. Soualmia LF, Dahamna B, Thirion B, Darmoni SJ. Strategies for health information retrieval. Stud Health Technol Inform 2006; 124: 595-600.

15. Darmoni SJ, Thirion B, Leroyt JP, Douyère $M$, Lacoste B, Godard $C$, et al. A search tool based on "encapsulated" $\mathrm{MeSH}$ thesaurus to retrieve quality health resources on the internet. Med Inform Internet Med 2001; 26: 165-178.

16. Shahar E. A popperian perspective of the term "evidencebased medicine». J Eval Clin Pract 1997; 3: 109-116.

17. Osborn J. Observation, Sherlock Holmes, and Evidence Based Medicine. Med Secoli 2002; 14: 515-527.

18. Gheith ME, Siam GA, de Barros DS, Garg SJ, Moster MR. Role of intravitreal bevacizumab in neovascular glaucoma. J Ocul Pharmacol Ther 2007; 23: 487-491.

19. Kelkar AS, Kelkar SB, Kelkar JA, Nagpal M, Patil SP. The use of intravitreal bevacizumab in neovascular glaucoma: a case report. Bull Soc Belge Ophthamol 2007; 303: 43-45.

20. Yazdani S, Hendi K, Pakravan M. Intravitreal bevacizumab (Avastin) injection for neovascular glaucoma. J Glaucoma 2007; 16: 437-439.

21. Chilov MN, Grigg JR, Playfair TJ. Bevacizumab (Avastin) for the treatment of neovascular glaucoma. Clin Experiment Ophthalmol 2007; 35: 494-496.

22. Batioglu F, Astam N, Ozmert E. Rapid improvement of retinal and iris neovascularisation after a single intravitreal bevacizumab injection in a patient with central retinal vein occlusion and neovascular glaucoma. Int Ophthalmol 2008; 28: 59-61.
23. Vatavuk Z, Bencic G, Mandic Z. Intravitreal bevacizumab for neovascular glaucoma following central retinal artery occlusion. Eur J Ophthalmol 2007; 17: 269-271.

24. Jonas JB, Spandau UH, Schlichtenbrede F. Intravitreal bevacizumab for filtering surgery. Ophthalmic Res 2007; 39: 121-122.

25. Iliev ME, Domig D, Wolf-Schnurrbursch U, Wolg S, Sarra GM. Intravitreal bevacizumab (Avastin) in the treatment of neovascular glaucoma. Am J Ophthalmol 2006; 142: 1054-1056.

26. Mason JO 3rd, Albert MA Jr, Mays A, Vail R. Regression of neovascular iris vessels by intravitreal injection of bavacizumab. Retina 2006; 26: 839-841.

27. Silva Paula J, Jorge R, Alves Costa R. Rodrigues Mde L, Scoutt IU. Short-term results of intravitreal bevacizumab (Avastin) on anterior segment neovascularization in neovascular glaucoma. Acta Ophthalmol Scand 2006; 84: 556-557.

28. Grisanti $S$, Biester $S$, Peters $S$, Tatar $O$, Ziemssen $F$, Bartz-Schmidt $K U$, et al. Intracameral bevacizumab for iris rubeosis. Am J Ophthalmol 2006; 142: 158-160.

29. Kahook MY, Schuman JS, Noecker RJ. Intravitreal bevacizumab in a patient with neovascular glaucoma. Ophthalmic Surg Lasers Imaging 2006; 37: 144-146.

30. Davidorf FH, Mouser JG, Derick RJ. Rapid improvement of rubeosis iridis from a single bevacizumab (Avastin) injection. Retina 2006; 26: 354-356.

31. Avery RL. Regression of retinal and iris neovascularization after intravitreal bevacizumab (Avastin) treatment. Retina 2006; 26: 352-354.

32. Oshima Y, Sakaguchi H, Gomi F, Tano Y. Regression of iris neovascularization after intravitreal injection of bevacizumab in patients with proliferative diabetic retinopathy. Am J Ophthalmol 2006; 142: 155-158.

33. Al-Aswad L. Another role for Avastin? Neovascular glaucoma. Rev Ophthalmol 2006; 13: 6.

34. Wakabayashi T, Oshima Y, Sakaguchi H, Ikuno Y, Miki A, Gomi $F$, et al. Intravitreal bevacizumab to treat iris neovascularization and neovascular glaucoma secondary to ischemic retinal diseases in 41 consecutive cases. Ophthalmology 2008; 115: 1571-1580.

35. Ehlers JP, Spirn MJ, Lam A, Sivalingam A, Samuel MA, Tasman W. Combination intravitreal bevacizumab/panretinal photocoagulation versus panretinal photocoagulation alone in the treatment of neovascular glaucoma. Retina 2008; 28: 696-702.

36. Chalam KV, Gupta SK, Grover S, Brar VS, Agarwal S. Intracameral Avastin dramatically resolves iris neovascularization and reverses neovascular glaucoma. Eur J Ophthalmol 2008; 18: 255-262.

37. Kitnarong N, Chindasub P, Metheetrairut A. Surgical outcome of intraviteal bevacizumab and filtration surgery in neovascular glaucoma. Adv Ther 2008; 25: 438-443.

38. Cheng JY, Wong DW, Ang CL. Intraocular avastin (bevacizumab) for neovascularization of the iris and neovascular glaucoma. Ann Acad Med Singapore 2008; 37: 72-74.

39. Cornish KS, Ramamurthi S, Saidkasimova S, Ramaesh K. Intravitreal bevacizumab and augmented trabeculectomy for neovascular glaucoma in young diabetic patients. Eye 2008; 2: doi:10.138/eye.2008.113.

40. Sothornwit N. Intravitreal bevacizumab for ahmed glaucoma valve implantation in neovascular glaucoma: a case report. J Med Assoc Thail 2008; 91: S162-S165. 
41. Bianciotto C, Shields CL, Kang B, Shields JA. Treatment of iris melanoma and secondary neovascular glaucoma using bevacizumab and plaque radiotherapy. Arch Ophthalmol 2008; 126: 578-579.

42. Avery RL, Pearlman J, Pieramici DJ, Rabena MD, Castellarin AA, Nasir MA, et al. Intravitreal bevacizumab (Avastin) in the treatment of proliferative diabetic retinopathy. Ophthalmology 2006; 113: 1695 1705.

43. Groover S, Gupta SK, Sharma RK, Brar VS, Chalam KV. Intracameral Bevacizumab Effectively Reduces Aqueous VEGF Levels in Neovascular Glaucoma. Br J Ophthalmol 2008; 10 [Epub ahead of print].

44. Martinez-Carpio PA, Navarro Moreno MA. Growth factors, cell damage, cyclin-dependent kinases and their inhibitors: relevance in molecular pathology of human cancer. Med Clin (Barc) 2003; 120: 265-271.
45. Tamm ER, Fuchshofer R. What increases outflow resistance in primary open-angle glaucoma? Surv Ophthalmol 2007; 52: S101-S104.

46. Iyengar L, Patkunanathan B, Lynch OT, McAvoy JW, Rasko JE, Lovicu FJ. Aqueous humor and growth factor-induced lens cell proliferation is dependent on MAPK/ERK1/2 and Akt/p13-K signalling. Exp Eye Res 2006; 83: 667-678.

47. Tu KL, Kaye SB, Sidaras G, Taylor W, Shenkin A. Effect of intraocular surgery and ketamine on aqueous and serum cytokines. Mol Vis 2007; 13: 1130-1137.

48. Vela JI, Galán A, Fernández E, Romera M, Torres JJ. Uveítis anterior y artritis idiopática juvenil. Arch Soc Esp Oftalmol 2003; 78: 561-565.

49. Simón G. Trabeculoplastia con dos tipos de láser en gato, monos y humano: estudio morfológico con microscopía electrónica de barrido. Arch Soc Esp Oftalmol 2006; 81: 527-536. 